\title{
Apollo Lab Diagnostics - A Revolutionary System to Improve Healthcare
}

Molecular diagnostics, the recent addition in Lab, is a collection of techniques used to analyze biological markers in the genome (the individual's genetic code) and proteome (how their cells express their genes as proteins) by applying molecular biology to medical testing. The recent rise of molecular biology has revolutionized the diagnosis and treatment of diseases. Modern test methods, based on the detection of DNA and RNA, offer many advantages over traditional methods. The new procedures can detect viruses, bacteria and genetic variations faster and much more accurately. Treatments can be tailored better to individual patients depending on person's genetic profile.

There are global reports of increasing incidence of non-tuberculous mycobacterium (NTM) causing variety of illness. However, it was not reported earlier in Bangladesh probably due to lack of sensitive methods to discriminate from MTB. In this volume M. Rahman et al. has reported its existence from variety of clinical specimens by PCR. This report is important in two respect; firstly, conventional methods cannot identify NTM and misread it as MTB. Secondly, Anti-TB therapy do not work well on NTM and needs different drugs to treat.

Adoption of multiplex PCR to identify system wise pathogen reduces cost of tests significantly. On the other hand, its use in hematological cancer categorizes patients need risk-adapted therapy. Further, introduction of flow cytometry in sub-typing of hematological malignancy and immunohistochemistry in solid cancer is playing key role in cancer diagnosis and giving Apollo Lab Medicine a specialized impression in the country.

Recent addition of Vitek 2 culture system in microbiology has made faster identification of bacteria and fungus at species level and knowing resistance with MIC will help clinicians for appropriate selection of antibiotic with right dosages.

Apollo Transfusion medicine is successfully doing Leukapheresis, TPE, Stem cell apheresis and Platelet apheresis and capable of supplying all types of blood components round the clock which has made the management of hematology patients easier.

Recent innovations in medical diagnostic technology underscore the role of diagnostic tests as a cornerstone of our health care system. Advanced molecular diagnostics, in particular, are helping provide more targeted care and treatment and are paving the way for personalized medicine. Next Generation Sequencing (NGS) technology will play revolutionary role in personalized medicine. Tertiary care givers should plan accordingly so that right medicine can be given to right patient.

Dr. Md. Mizanur Rahman

Consultant

Molecular Diagnostics

Apollo Hospitals Dhaka 